\title{
New robot navigation algorithm for arbitrary unknown dynamic environments based on future prediction and priority behaviour
}

\begin{abstract}
This study focuses on existing drawbacks and inefficiencies of the available path planning approaches within unknown dynamic environments. The drawbacks are the inability to plan under uncertain dynamic environments, non-optimality, failure in crowded complex situations, and difficulty in predicting the velocity vector of obstacles. This study aims (1) to develop a new predictive method to avoid static and dynamic obstacles in planning the path of a mobile robot in unknown dynamic environments in which the obstacles are moving and their speed profiles are not pre-identified, to find a safe path and to react rapidly and (2) to integrate a decision-making process with the predictive behavior of the velocity vector of obstacles by using the sensory system information of the robot. Information on the locations, shapes, and velocities of static and dynamic obstacles is presumed to be unavailable. Such information is determined online using rangefinder sensors. Thus, the robot recognizes free directions that lead it toward its destination and keep it safe and prevent collision with obstacles. Extensive simulations confirm the efficiency of the suggested approach and its success in handling complex and extremely dynamic environments that contain various obstacle shapes. Findings indicate that the proposed method exhibits attractive features, such as high optimality, high stability, low running time, and zero failure rates. The failure rate is zero for all test problems. The average path length for all test environments is 16.51 with a standard deviation of 0.49 , which provides an average optimality rate of $89.79 \%$. The average running time is $4.74 \mathrm{~s}$ (the standard deviation is 0.26 ).
\end{abstract}

Keyword: Dynamic environment; Navigation; Obstacle avoidance; Path planning; Robotics 